\title{
Hernia interna secundaria a defecto del ligamento ancho*
}

\author{
Drs. JOSÉ HOLA B. ${ }^{1}$, RODRIGO AZOLAS M. ${ }^{1}$, MARIO ABEDRAPO M. ${ }^{1}$, VÍCTOR AVILLO ${ }^{2}$, \\ MARCOS SOBRON B. ${ }^{2}$, ROGELIO GARRIDO C. ${ }^{1}$, GUNTHER BOCIC A. ${ }^{1}$, ALVARO SANHUEZA S. ${ }^{3}$
}

1 Departamento de Cirugía.

2 Interno de Medicina.

3 Departamento de Radiología.

Hospital Clínico Universidad de Chile.

Santiago, Chile.

\section{Hydatid bronchobiliary fistula}

Paciente mujer de 24 años, sin antecedentes médicos, obstétricos ni quirúrgicos previos. Presenta cuadro de 24 horas de evolución sugerente de obstrucción intestinal. La tomografía computada de abdomen y pelvis (Figuras 1 y 2) informa signos de obstrucción de asas de intestino delgado, cuya etiología podría corresponder como primera posibilidad a endometriosis. Escaso líquido peritoneal libre.

Se realiza laparotomía exploradora, encontrándose una hernia interna a través de un defecto del parametrio izquierdo por donde protruía un asa de íleon distal, la que presentaba signos de isquemia (Figuras 3 y 4). Se procede a liberar el segmento de asa comprometida, recuperando una coloración normal y su peristaltismo. El defecto del parametrio se suturó con Vicryl 3/0 a puntos separados. La paciente evoluciona bien y es dada de alta al segundo día postoperatorio.

La obstrucción intestinal es una patología frecuente de ver en los servicios de urgencia, representando aproximadamente el $12-16 \%$ de los ingresos ${ }^{1}$. Las hernias internas como causa de obstrucción intestinal son extremadamente raras, ocurriendo en menos del $1 \%$ de los casos, más aún, si la causa es una herniación a través de un defecto del ligamento ancho, debido a que sólo corresponden a un 4-5\% de las hernias internas ${ }^{2}$. En una revisión extensa de la literatura se han encontrado sólo 73 casos de herniación a través de un defecto del ligamento ancho desde que Quain en 1861 describiera el primer caso ${ }^{3}$. En la mayoría de los reportes fue el íleon el segmento intestinal herniado ${ }^{4}$, sin embargo, otras estructuras como colon, ovario, omento, apéndice y uréter también han sido mencionados ${ }^{5}$. El ligamento ancho del útero está formado por peritoneo cubriendo sus dos superficies. Un defecto puede involucrar una o ambas superficies peritoneales y frecuentemente ocurre en ambas. Dentro de los factores etiológicos a ser considerados se incluyen: traumatismo del parto, anomalías congénitas, enfermedad inflamatoria pelviana y, en el pasado, la cirugía de Webster-Baldy como tratamiento de la retroversión uterina, que creaba aperturas en el ligamento ancho ${ }^{6}$.

La detección preoperatoria de una herniación a través de un defecto en el ligamento ancho puede ser difícil debido a la ausencia de un cuadro clínico característico, sin embargo, reportes recientes indican que la tomografía computada de abdomen y pelvis puede sugerir un diagnóstico correcto, aunque no preciso ${ }^{7}$.

\footnotetext{
* Recibido el 16 de junio de 2009 y aceptado para publicación el 27 de julio de 2009.

Correspondencia: Dr. Rodrigo Azolas M.

Santos Dumont 999, Santiago, Chile.

E-mail: rodrigoazolas@gmail.com
} 


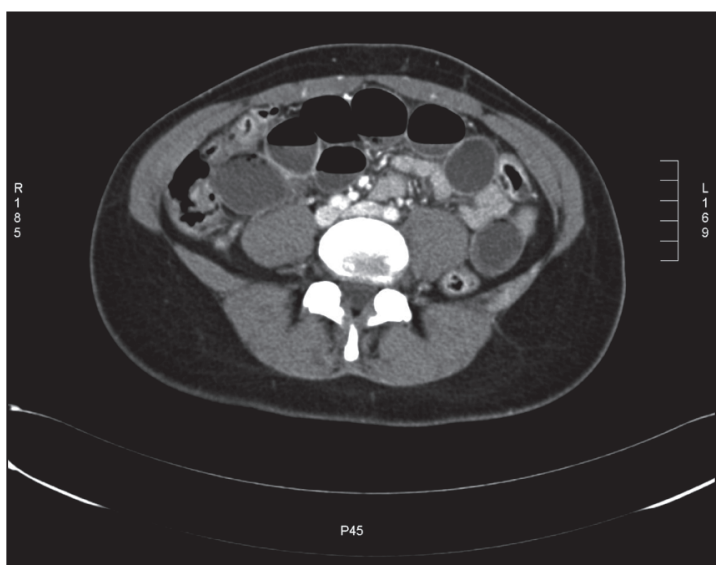

Figura 1. TAC que muestra asas dilatadas de intestino delgado.

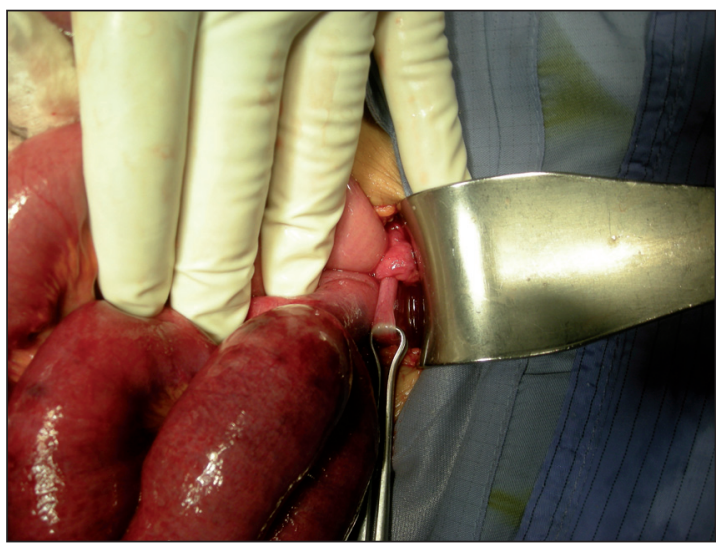

Figura 3. Asa dilatada en orificio herniario.

\section{Referencias}

1. Wetch J. General consideration and mortality in bowel obstruction. In: Welch JP, ed Bowel obstruction: differential diagnosis and clinical management. Philadelpia: WB Saunders 1990: 59-95.

2. Fukuoka M, Tachibana S, Harada N, Saito H. Strangulated herniation through a defect in the broad ligament. Surgery 2002; 131: 232-233.

3. Garcia-Oria M, Inglada J, Domingo J, Biescas J, Ching C. Journal of Laparoendoscopic \& Advanced Surgical Techniques 2007; 17: 666-668.

4. Rabushka SE. Colon hernia through a hiatus in the

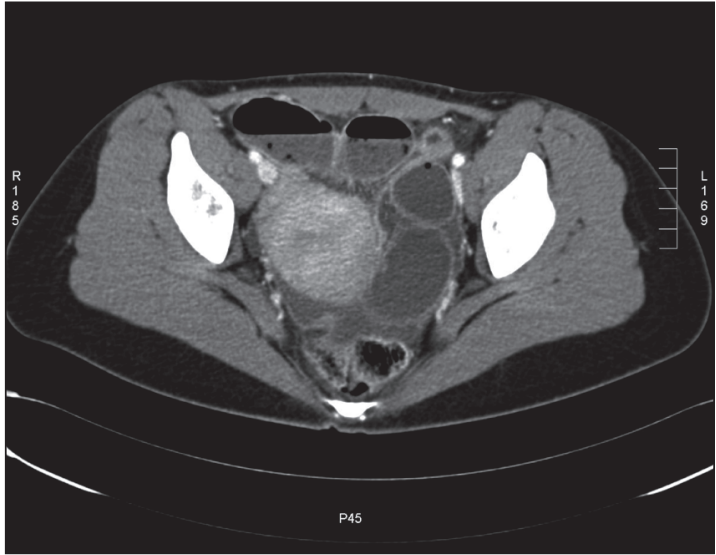

Figura 2. TAC que muestra asas dilatadas de intestino delgado.

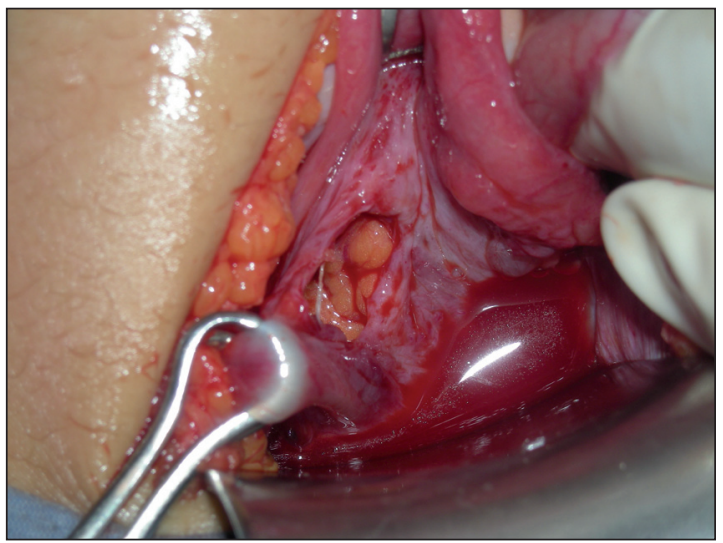

Figura 4. Orificio herniario.

broad ligament: report of a case and review of the literature. Obstet Gynecol 1968; 31: 261-265.

5. Slezak FA, Schlueter TM. Hernia of the broad ligament of the uterus. In: Nyhus LM, Codon RE. Hernia. $3^{\text {rd }}$ Edition. Philadelphia: J.B. Lippincott, 1989: 311-316.

6. Hunt AB. Fenestrae and pouches in the broad ligament as an actual potential cause of strangulated intra-abdominal hernia. Surg Gynecol Obstet 1934; 58: 906.

7. Suzuki M, Takashima T, Funaki H, Uogishi M, Isobe T, Kanno S. Radiologic imaging of a herniation of the small bowel through a defect in the broad ligament. Gastrointest Radiol 1986; 11: 102-110. 